\title{
Changing the Culture and Attitude: Supporting Women Studying Mining Engineering
}

\author{
Andrea Brickey ${ }^{1 *}$, Kelli McCormick ${ }^{1}$, Paula Jensen ${ }^{2}$, Michael West ${ }^{3}$ \\ ${ }^{1}$ Department of Mining Engineering and Management, South Dakota School of Mines and Technology, Rapid City, SD, \\ USA \\ ${ }^{2}$ Department of Industrial Engineering, South Dakota School of Mines and Technology, Rapid City, SD, USA \\ ${ }^{3}$ Department of Materials and Metallurgical Engineering, South Dakota School of Mines and Technology, Rapid City, SD, \\ USA
}

\begin{abstract}
Attracting and retaining women studying STEM fields can be difficult, but even more so in very male-dominated industries such as mechanical engineering, metallurgical engineering, and mining engineering. South Dakota School of Mines and Technology is an engineering and science research university located in the state of South Dakota, United States of America. Researchers at the university have developed a program, funded by a NSF S-STEM grant, which provides financial support, mentoring, and programmatic activities to approximately 30 female students. In this paper, we will discuss curriculum changes, e.g., classroom environment, project-based learning, made by the program's mining engineering faculty. We will also present lessons learned and ideas for future curriculum modifications.
\end{abstract}

Keywords: mining engineering, curriculum, undergraduate education, culture and attitude

\section{Introduction}

In 2015, the Department of Mining Engineering and Management (MEM) at the South Dakota School of Mines and Technology (SD Mines) was invited to participate in a National Science Foundation S-STEM grant, called Culture and Attitude (C\&A), with the purpose of recruiting and retaining women studying various engineering disciplines with low female enrollment. The goal of the program is to retain and support academically talented women, with financial need, seeking degrees in five STEM fields; civil, industrial, mechanical, metallurgical, and mining engineering. The program has been active at SD Mines since 2010, and originally focused on three disciplines, industrial, mechanical and metallurgical engineering. Mining and civil engineering were added to the program in 2015 and this paper is intended to review the ideas implemented and curriculum changes made in the mining department as part of this program and to discuss some of rewards and challenges.

According to the 2015-2016 report, Engineering by the Numbers (Yoder 2012), there were 308 mining engineering bachelor's degrees awarded in the United States that academic year, the lowest number of the reported disciplines. Of the 308 mining engineering graduates, only $14.3 \%$ (44) were women.

As of April 2018, the MEM department at SD Mines has 83 undergraduate students. Of those students, 12 are female or $14.5 \%$. This sample size makes it extremely difficult to provide accurate measurements of the S-STEM program's effectiveness, but the authors believe that over the life of the C\&A program, the long-term impacts will become more evident and quantifiable. This paper will discuss the Culture and Attitude program, curriculum changes being made in the MEM department, and other efforts being made to increase matriculation of women studying mining engineering at SD Mines.

\section{Culture and Attitude Program}

The primary goal of the Culture \& Attitude program is to increase the number of women graduating with engineering degrees. The C\&A program aims to achieve that goal by providing financial support, mentoring, and technical and professional development activities for the scholars. Students have access to a faculty member for one-on-one mentoring in addition to monthly group activities. These activities provide an opportunity for networking and social interactions. Monthly activities include 3-D printing, industry tours, and professional development topics, among others.

All C\&A students are required to meet with a mentor once a semester, but mentoring is available on a more frequent basis if desired by the student. Many of the scholars are also active in the Women in Science and Engineering (WiSE) program, which provides numerous outreach and social activities for students.

* Corresponding Author: Andrea Brickey, Email: andrea.brickey@ sdsmt.edu, phone: +01 6053941275

Copyright @ 2018 Canamaple Academia Services, http://press.camdemia.ca

DOI: 10.15273/ijge.2018.03.027 
In addition to faculty and staff support, industry has shown substantial support for the program. For example, in 2017, students went on an overnight trip to dine with senior management of a large surface coal mine and tour the mine and maintenance operations (Figure 1). These activities serve to provide student exposure to industry and to illustrate the importance of engineering and the rewarding nature of problem solving.

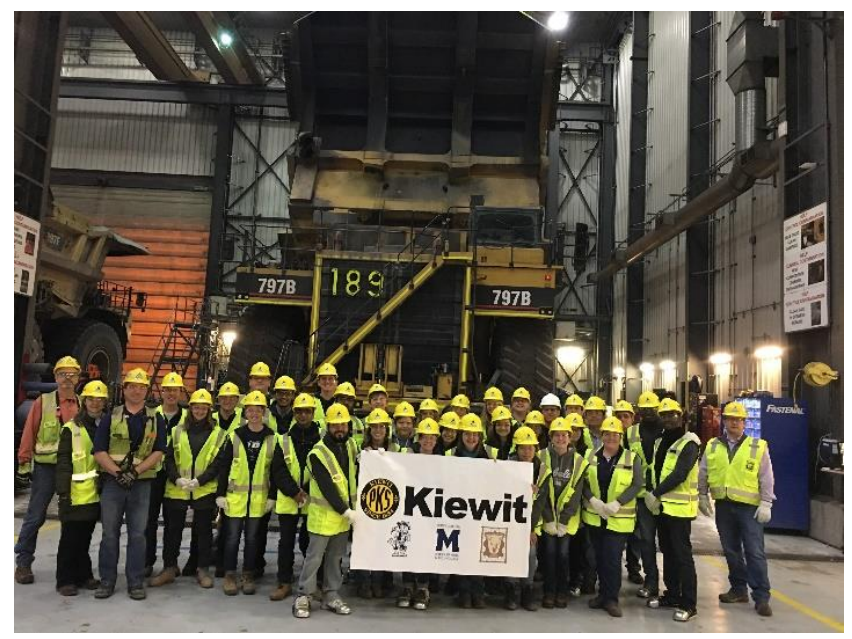

Figure 1 Photo of students, including many Culture and Attitude scholars at a large surface coal mine in Gillette, Wyoming, USA. (Photo by Paula Jensen)

In determining how to effectively change the culture at SD Mines to appreciate different thinking styles, an analysis was conducted to better understand the typology of engineering students (Karlin \& Kellogg 2009). The results of this analysis showed that women, on average, have different thinking styles when compared to their male counterparts. This analysis used the Herrmann Brain Dominance Inventory (HDBI) which classifies thinking styles into four categories; analytical, practical, relational, and experimental. The typical engineering curriculum caters to the analytical thinking style, which fits most students graduating with STEM degrees. While female students tend to fall more broadly into the four quadrants prior to graduation, those falling outside of the analytical quadrant are less likely to graduate with a STEM degree. A primary focus of the C\&A program (Kellar et al 2015) is to create classroom environments and curriculum modifications that expand beyond the analytical thinking style, which is in line with expected industry needs as described in The Engineer of 2020 (Clough 2004).

Another standard that has been of significant benefit when building curriculums and planning class activities are the twelve best practice factors identified by Burke \& Mattis (2007). These factors enable faculty to provide support to women and minorities in STEM. Of the twelve factors Burke identified, the C\&A program focuses on six of these (bold below).

(1) Create a classroom environment that makes it easy to ask questions;

(2) Use group projects that foster cooperative learning;
(3) Show how STEM knowledge is used in industry;

(4) Communicate the use of STEM to broader life and social issues;

(5) Choose projects that make applications clear and that build on the student's previous experiences;

(6) Use various teaching methods to appeal to different learning styles;

(7) Offer remedial classes;

(8) Use inclusive language and examples;

(9) Address teachers' sexist beliefs and stereotypes;

(10) Grade assignments blind to the identity of students.

(11) Use information such as web documents to encourage students to take STEM courses;

(12) Offer summer school courses to help talented precollege students to overcome deficiencies in their backgrounds and build confidence in their skills in STEM.

\section{Curriculum Modifications}

Faculty in the MEM department began by evaluating the classroom environment and course curriculum to identify several focus areas based on the Burke factors. With a focus on improving discussion and developing more freeform dialogue, the faculty asked students, when posed a question, to consult another student before responding to the question. This gave the students an opportunity to think about the question, to build their confidence, and to encourage more participation. As the students practiced this during the first few classes, faculty emphasis on this process was less necessary as the students began to feel more comfortable and dialogue became more natural. In another class, the faculty established quizzes that allowed for group discussion to solve the problem.

Many of the upper level courses already included project-based learning, so the faculty focused on moving the projects from a strictly analytical approach to incorporating more creative problem solving. An example of this was implemented in the explosives engineering course, where students were given a general set of parameters and then asked to make reasonable assumptions in the context of a 'story'. Then the assumptions were explained as the solution was presented. For example, the student might assume that his or her blast design problem was at a mine located in a rain forest or a desert and must compete the project using appropriate parameters. As another example, the faculty encouraged students to create a story line behind the data and analysis reported in memos for the class.

To show how STEM knowledge is used in industry, the mining faculty invited several guest speakers to the campus, but also provided many real-world examples during the instructional period. It was beneficial in this case to have several faculty with in-depth mining industry experience.

The faculty made a special effort to show how STEM knowledge and problem-solving abilities are used to benefit society. This was accomplished by bringing in current issues and topical focus items for discussion. It was especially important to help the students see beyond the obvious to the greater positive impact mining has on a local, national and global scale. 
As the students increase in knowledge and ability, the projects assigned to them become more complex and require a greater ability to synthesize knowledge across disciplines. An example of this is in the Senior Capstone course where students were assigned a real-world project with actual data from an operating mine. They were asked to bring in their knowledge from their course work, such as ventilation, mine design, reclamation and rock mechanics to develop a solution that will be presented to faculty and industry representatives.

The faculty also expanded upon traditional teaching methods and implemented new technologies and approaches with excellent success. One example is 'video Friday' in explosives engineering, where short videos of various blasting techniques would be shown and then the class would provide analysis.

\section{Evaluation}

A survey was recently conducted to evaluate the MEM undergraduate students' preferences and perception of learning. The survey was administered in March of 2018 and was sent to the undergraduate students in the MEM department. Of the 83 students, 41 responded to the survey and of those 10 identified as female. The data is presented as a total of all students and again with only the female students. The questions were phrased to gain some insight into the students' learning style preference, along with the effectiveness of those learning styles. The survey asked the students to rate the statement from 1 to 5 , representing the following responses; strongly disagree (1), disagree (2), neutral (3), agree (4), strongly agree (5). The survey consisted of the following statements;

(a) I prefer classroom discussions, group quizzes and group responses to questions versus individual quizzes and individual responses to directed questions.

(b) I learn more from classroom discussions, group quizzes and group responses to questions versus individual quizzes and individual responses to directed questions.

(c) I prefer group work versus individual projects.

(d) I learn more with group work versus individual projects.

(e) I prefer to develop my own approach to a project versus having a list of directions.

(f) I learn more when I am allowed to approach a project in my own way versus having a list of directions.

Two final questions were asked with the options of yes, no, and most of the time, but still needs improvement. The final questions are as follows:

(a) Do you feel that the MEM department is welcoming to your gender?

(b) Do you feel that MEM classes (classroom environment) are welcoming to your gender?

The results of the first six questions for all respondents are shown in Figures 2, 3, and 4. The results from only female respondents are shown in Figures 5, 6, and 7. When asked if they prefer classroom discussions, group quizzes and group responses, many of the respondents indicated neutral to positive agreement as shown in Figure 2. The female respondents also indicated mostly neutral to positive agreement with the statement.

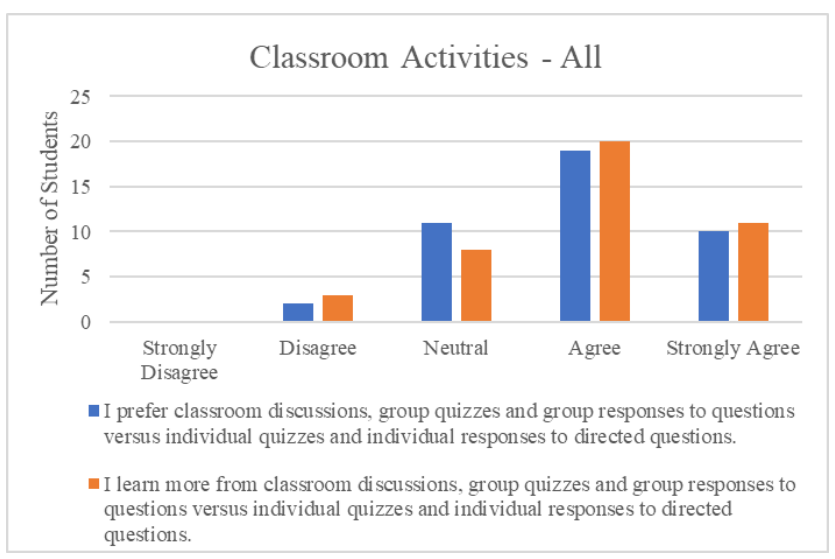

Figure 2 Survey results from all respondents to classroom activities

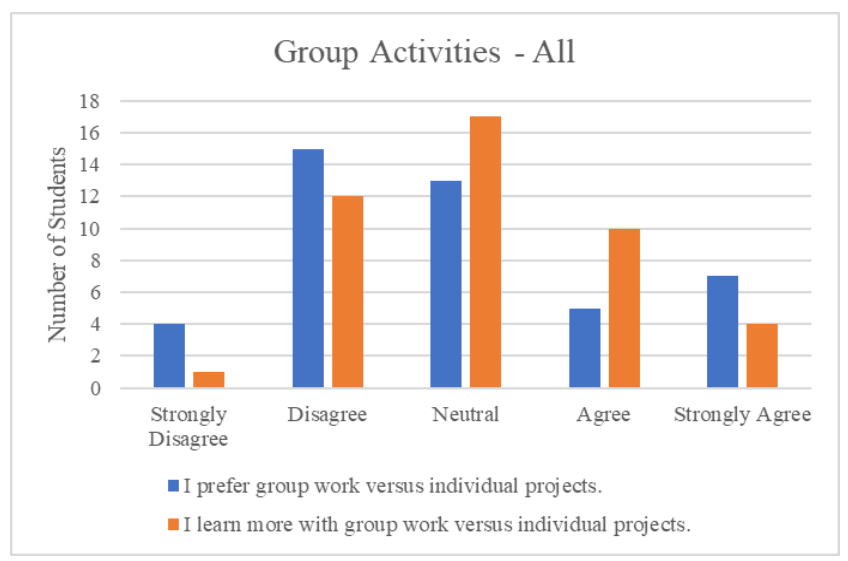

Figure 3 Survey results from all respondents to group activities

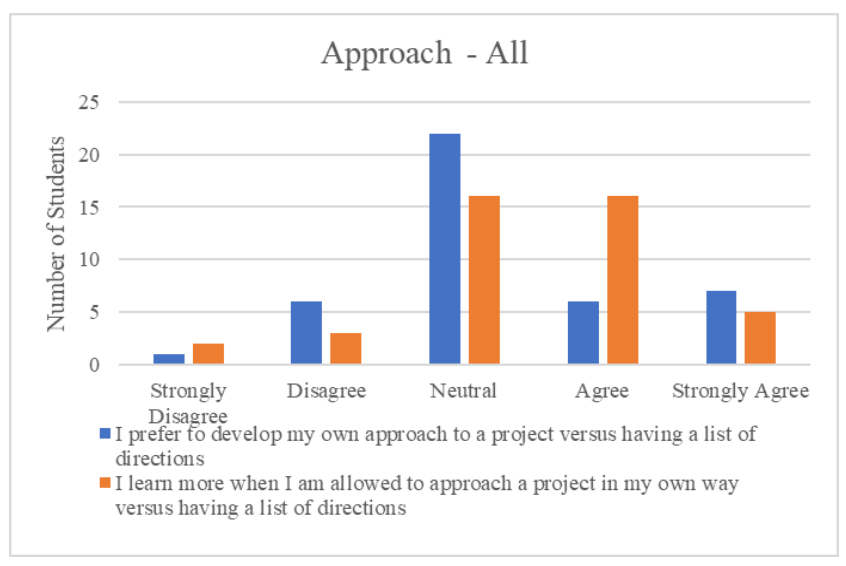

Figure 4 Survey results from all respondents to approach

Given the increasing importance of understanding social context and ability to work in teams as outlined in the Engineer of 2020, the survey results regarding whether students prefer and learn from group work versus individual work provide some interesting results. Students indicated neutral to negative when asked if they prefer group work, while they provide a neutral to positive response when asked if they learn more from group work. Although these 
are preliminary results, it will be interesting to assess the change in student attitudes and perceptions towards group and team assignments in the future.

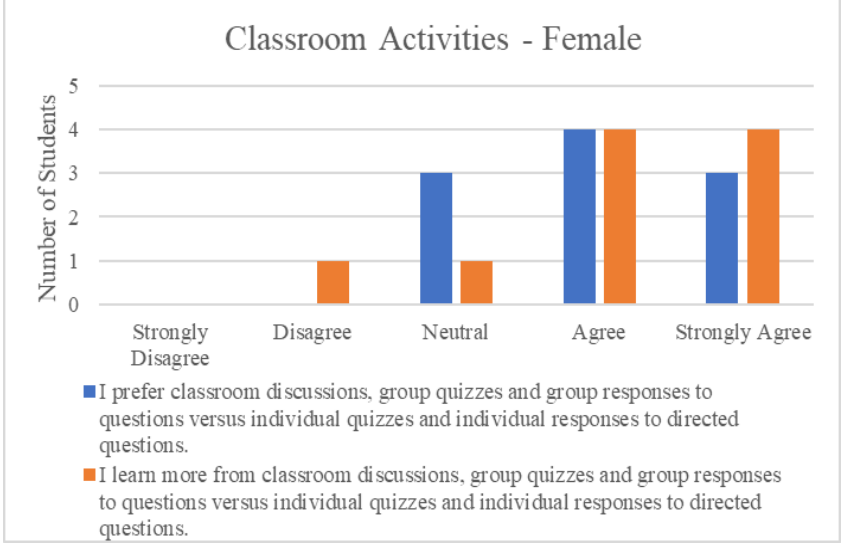

Figure 5 Survey results from female respondents to classroom activities

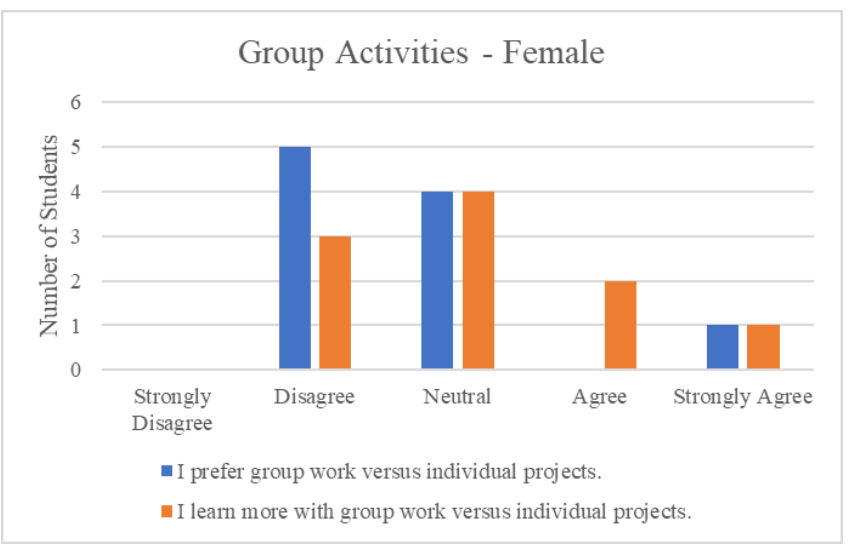

Figure 6 Survey results from female respondents to group activities

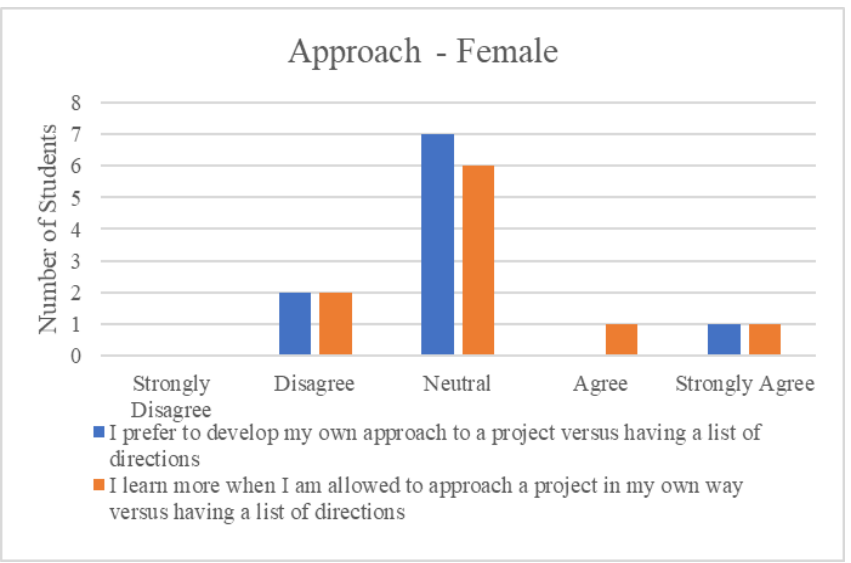

Figure 7 Survey results from female respondents to approach

With respect to projects, most students indicated that they prefer and learn more when allowed to develop their own approach to a project versus a list of directions. When isolating the female responses, the majority were neutral.

The final two questions ask the student if the MEM department was welcoming and if the classroom environment for MEM courses were welcoming to their gender. All 41 respondents answered 'yes' to the question of the MEM department being welcoming to their gender. One student, a female, did respond that the classroom environment was welcoming 'most of the time, but could use improvement' while all others responded 'yes'.

Considering the small sample size, it is difficult to make conclusive remarks on the results; however, the data gathered will be used to provide a baseline for future comparisons.

\section{Conclusions}

Some broad conclusions that we can draw from this survey are:

(1) most of the MEM women students are neutral or even dislike classroom or group discussions and work, but feel they learn more through this process (Figures 5 and 6). No significant difference between the MEM students, as a whole, (Figures 2 and 3) and women students only is apparent in these categories, though this may be a function of the low sample size;

(2) most of the MEM women students are neutral or somewhat dislike developing their own approach to a project or problem (Figure 7) and are neutral in feeling that they learn more through this process. Response to this question from all MEM students (Figure 4) suggests that more male MEM students feel they learn more by developing their own approach to a project than female students.

The Mining Engineering and Management Department at South Dakota School of Mines and Technology is dedicated to increasing the number of women pursuing a degree in mining engineering. The faculty are actively working to incorporate novel pedagogical methods to help retain and graduate a diverse group of mining engineers.

The survey information collected will serve as a baseline for future evaluation of the curriculum changes within the MEM department. Future work will involve developing a survey to give to students at the beginning of a course and at the end of the course to determine the impacts of the curriculum changes. The focus of future curriculum changes includes incorporating online, interactive quiz platforms, i.e., Kahoot, and soliciting industry professionals to mentor students as part of the senior capstone course.

\section{Acknowledgement}

This research project is supported by the National Science Foundation Division of Undergraduate Education S-STEM Award 1564837. The authors also extend appreciation to the South Dakota School of Mines and Technology and the Department of Mining Engineering and Management.

\section{References}

Burke, R.J. and M.C. Mattis, 2007. Women and minorities in science, technology, engineering, and matematics: Upping the numbers. Edward Elgar Publishing. 
Clough, G.W., 2004. The engineer of 2020: Visions of engineering in the new century. National Academy of Engineering, Washington, DC.

Karlin, J. and S. Kellogg, 2009. Metrics and the holistic learner. Proceedings of the Research in Engineering Education Symposium, Palm Cove, QLD, pp 1-9.
Kellar, J., J. Karlin, D. Medlin and S. Kellogg, 2015. SSTEM: Culture and Attitude -- Innovative Partnerships for Success. NSF Grant Applicaiton.

Yoder, B.L., 2012. Engineering by the Numbers. American Society of Engineering Education. 